\title{
Induction of Hypopituitarism Following Ipilimumab/ Nivolumab Therapy Followed by Radiation in the Treatment of Metastatic Scalp Melanoma
}

\author{
Joshua K. Salabei, MD, PhD, 'Dhaval Upadhyay, MD,' Sripal A. Padam, MD
}

\section{Abstract}

\section{Description}

Immune checkpoint inhibitors $(\mathrm{ICl})$ are antagonistic antibodies that block specific immune checkpoint molecules, such as cytotoxic T-lymphocyte-associated antigen 4 (CTLA-4), programmed cell death protein (PD-1) and its ligand PD-L1. With FDA approval, the use of these checkpoint inhibitors has led to long-lasting tumor responses. However, by stimulating the immune system, checkpoint inhibitors can cause immune-related adverse events involving the endocrine organs, among others. Pituitary dysfunction (hypophysitis) leading to secondary adrenal insufficiency, or primary adrenal insufficiency caused by immune checkpoint inhibitors, have been documented. In this report, we present a case of a 70-year-old man with scalp melanoma with metastasis to the neck lymph nodes who remained asymptomatic 2 weeks after treatment completion with ipilimumab/nivolumab. However, he became lethargic, lost his appetite and was unable to perform activities of daily living following the initiation of radiation therapy to his neck. After hospitalization, he was found to have hyponatremia, hypocortisolism and hypopituitarism. He was treated with hydrocortisone, which lead to significant symptom improvement. His case suggests a dual-hit mechanism of injury to the pituitary caused by combined $\mathrm{ICl}$ and radiation therapies. Following $\mathrm{ICl}$ therapy, he was on the verge of pituitary dysfunction that fully materialized following a second insult. We suggest that for patients treated with ICls, particularly with ipilimumab/nivolumab, a washout period may be considered before starting other forms of therapies, such as radiation therapy to the head/neck regions. Otherwise, prophylactic low dose corticosteroids may be initiated in cases where radiation therapy must not be delayed.

\section{Keywords}

hypopituitarism; ipilimumab; nivolumab; melanoma; metastasis; neoplasms; immune checkpoint inhibitors/adverse effects; pituitary diseases; radiotherapy; hypophysitis; hyponatremia

\section{Introduction}

Ipilimumab, a human immunoglobulin $\mathrm{G}(\lg G) 1 \mathrm{~K}$ anti-cytotoxic T-lymphocyte antigen 4 (CTLA4) monoclonal antibody, and nivolumab, a human IgG4K anti-programmed cell death protein 1 (PD-1) monoclonal antibody, are approved by the FDA for the treatment of metastatic melanoma.' Despite the established benefits seen with these immune checkpoint inhibitors (ICls), their therapeutic effects are not isolated to tumor-specific T cells. As such, adverse events, collectively referred to as immune-related adverse events (IRAEs), remain a major concern with their use..$^{2,3}$
IRAEs can affect any organ or tissue, with a predilection to certain organs like the skin, intestine, liver, lungs and endocrine glands. ${ }^{3,4} \mathrm{Hy}$ pophysitis leading to adrenal insufficiency has been documented in patients treated with ICls, particularly in those treated with ipilimumab and nivolumab where the reported prevalence is about $12 \%$ and $0.5 \%$, respectively. ${ }^{5}$ Lately, many reports on various endocrinopathies have been published with secondary adrenal insufficiency resulting from hypopituitarism as one of the common IRAEs observed with the use of ICls. Hypopituitarism secondary to hypophysitis is, therefore, one of the dose-limiting

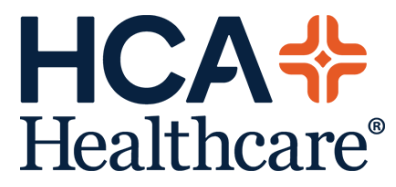

www.hcahealthcarejournal.com

(c) 2021 HCA Physician Services, Inc. d/b/a Emerald Medical Education
HCA Healthcare
Journal of Medicine 
complications, prompting recommendations to monitor pituitary function in patients undergoing treatments. ${ }^{6}$

For patients with head/neck cancers or with brain metastatic cancer successively treated with $\mathrm{ICls}$ and who did not experience any symptoms suggestive of hypopituitarism, follow-up radiation therapy to the head/neck within a given window could cause hypopituitarism leading to life-threatening adrenal insufficiency. Herein, we report the case of a 70-yearold man who received 4 rounds of treatment with ipilimumab/nivolumab for scalp melanoma with metastasis to sentinel lymph nodes. Follow-up radiation therapy to the head/neck 1 month after $\mathrm{ICI}$ therapy led to life-threatening hypopituitarism requiring hospitalization and treatment with hormone replacement therapy.

\section{Case Presentation}

A 70-year-old man with a history of malignant scalp melanoma and hypertension presented with complaints of 1 week of generalized weakness, poor appetite and decreased oral intake, inability to perform activities of daily living, multiple presyncopal episodes and a fall with associated head trauma on the day of presentation. About 6 months prior, the patient had a wide excision of his scalp melanoma. A biopsy of the sentinel node was positive for malignant cells, and the PET scan was positive for metastatic disease in the neck lymph nodes. His tumor was also BRAF positive. He was treated with 4 cycles of immunotherapy consisting of ipilimumab and nivolumab, lasting a total of 20 weeks. A follow-up PET scan showed progression of the disease to the neck lymph nodes. He was then started on radiation therapy, approximately 2 weeks after his last dose of ipilimumab/nivolumab. His presenting symptoms started within 24 hours after his first dose of radiation, prompting his visit to the hospital. His oncologist had also started him on dabrafenib (a BRAF inhibitor) and trametinib (an MEK inhibitor), which he started taking only after his initial dose of radiation, i.e., after his presenting symptoms had begun (see Figure 1 for a timeline of events).

On presentation, the patient was found to have a laceration on his scalp, secondary to the fall he sustained. His physical exam showed a lethargic man with a dull affect. His mucous membranes appeared dry and his lips chapped. His cardiac, lung and abdominal exams were unremarkable. No skin discoloration was noted. His cranial nerves were intact, and his muscular strength was equal bilaterally. However, he was unable to lift his hand above his head. His vital signs and initial labs are summarized in Table 1. A CT of the cervical spine did not show osseous injury, and a CT of the brain showed no lesions, hemorrhage or fluid collection. An MRI of the spine showed markedly enlarged bilateral suboccipital lymph nodes. Serum sodium and osmolarity of $118 \mathrm{mmol} / \mathrm{L}$ and $253 \mathrm{mosm} / \mathrm{Kg}$ were notable on his labs, as well as urine sodium and osmolality of $578 \mathrm{mosm} / \mathrm{Kg}$ and $17 \mathrm{mmol} / \mathrm{L}$. His sodium level was unresponsive to intravenous normal saline administration, fluid restriction or salt tablets. His home antihypertensive medicine (triamterene/HCTZ) was held. However, his sodium levels remained unaltered. Subsequent tests were significant for low levels of ACTH, TSH, FSH and LH. (Table 1) Cosyntropin

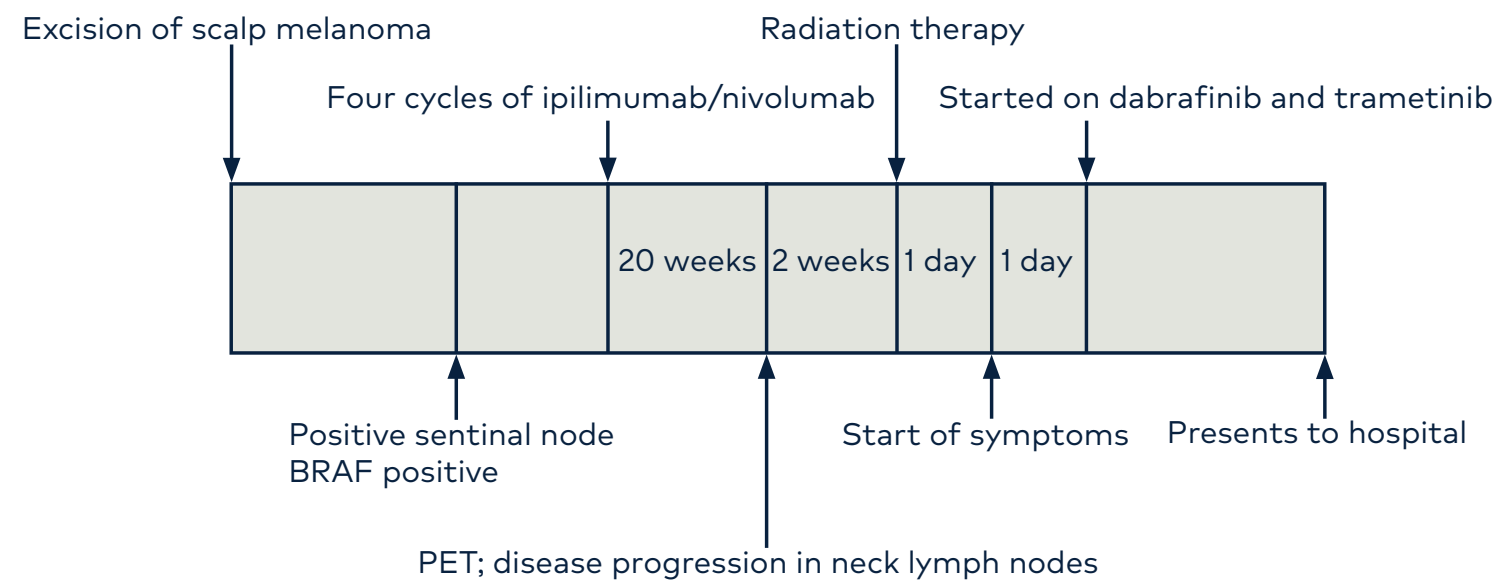

Figure 1. A timeline of events leading to presentation. 
Table 1. Pertinent Laboratory Data at Time of Presentation

Labs

\begin{tabular}{lcc}
\hline White blood cells & 6.5 & $\left(4.5-11.0\right.$ thou/mm $\left.{ }^{3}\right)$ \\
\hline Hemoglobin & 15.4 & $(12.0-15.0 \mathrm{~g} / \mathrm{dL})$ \\
\hline Platelet Count & 203 & $(150-450$ thousand/mm $)$ \\
\hline Sodium & 118 & $(136-145 \mathrm{mmol} / \mathrm{L})$ \\
\hline Potassium & 3.7 & $(3.5-5.1 \mathrm{mmol} / \mathrm{L})$ \\
\hline Serum osmolality & 253 & $(275-301 \mathrm{mOsm} / \mathrm{kg})$ \\
\hline BUN & 17 & $(7-18 \mathrm{mg} / \mathrm{dL})$ \\
\hline Creatinine & 0.84 & $(0.60-1.30 \mathrm{mg} / \mathrm{dL})$ \\
\hline Urine osmolality & 578 & $(50-1200 \mathrm{mOsm} / \mathrm{kg})$ \\
\hline Urine sodium & 17 & $(40-220 \mathrm{mmol} / \mathrm{L})$ \\
\hline Calcium & 8.5 & $(8.5-10.1 \mathrm{mg} / \mathrm{dL})$ \\
\hline AST & 54 & $(15-37 \mathrm{units} / \mathrm{L})$ \\
\hline ALT & 199 & $(12-78 \mathrm{units} / \mathrm{L})$ \\
\hline ALP & 70 & $(46-116 \mathrm{units} / \mathrm{L})$ \\
\hline ACTH & $<1.5$ & $(7.2-63 \mathrm{pg} / \mathrm{mL})$ \\
\hline FSH & 3.7 & $(0-200 \mathrm{mlnU} / \mathrm{mL})$ \\
\hline LH & 2.4 & $(0-200 \mathrm{mlnU} / \mathrm{mL})$ \\
\hline TSH & 0.19 & $(0.35-3.74 \mathrm{ulU} / \mathrm{mL})$ \\
\hline Free T4 & 0.8 & $(0.76-1.46 \mathrm{ng} / \mathrm{dL})$ \\
\hline Cortisol & 2.7 & $(8.0-14.7 \mathrm{pg} / \mathrm{dL})$ \\
\hline ADH & 10 & $(\mathrm{dL})$ \\
\hline
\end{tabular}

$\mathrm{BUN}=$ blood urea nitrogen; $\mathrm{AST}=$ aspartate aminotransferase; $\mathrm{ALT}=$ alanine aminotransferase; $\mathrm{ALP}=$ alkaline phosphatase; $\mathrm{ACTH}=$ adrenocorticotrophic hormone; $\mathrm{FSH}=$ follicle-stimulating hormone; $\mathrm{LH}=$ luteinizing hormone; $\mathrm{TSH}=$ thyroid-stimulating hormone; $\mathrm{ADH}=$ antidiuretic hormone

stimulation led to increased serum levels of ACTH. Suspicious of $\mathrm{ICl}$-induced hypophysitis leading to secondary adrenal insufficiency, an MRI of the brain was done, which showed no abnormalities. His clinical course improved dramatically after the initiation of hydrocortisone (10 mg/day) and levothyroxine $(25 \mu \mathrm{g} /$ day $)$. His sodium level was greater than $130 \mathrm{mmol} / \mathrm{L}$ at the time of discharge, (Figure 2) and he was discharged on a prescription of hydrocortisone and levothyroxine to follow up with his primary care physician and oncologist.

\section{Discussion}

Among the $\mathrm{ICl}$-induced endocrinopathies, $\mathrm{ICl}$-induced hypophysitis leading to secondary adrenal insufficiency and $\mathrm{ICl}$-induced primary adrenal insufficiency has been well documented. ${ }^{7}$ The onset of $\mathrm{ICl}$-induced endocrinopathy varies and can occur from weeks to months after immunotherapy ${ }^{5,6}$ Hypophysitis is a common adverse event with anti CTLA-4 antibody monotherapy (incidence of less than or equal to $10 \%$ at a dose of $3 \mathrm{mg} / \mathrm{kg}$ and up to $17 \%$ at 10 $\mathrm{mg} / \mathrm{kg}$ of ipilimumab therapy) and with combination ipilimumab/nivolumab (incidence less 


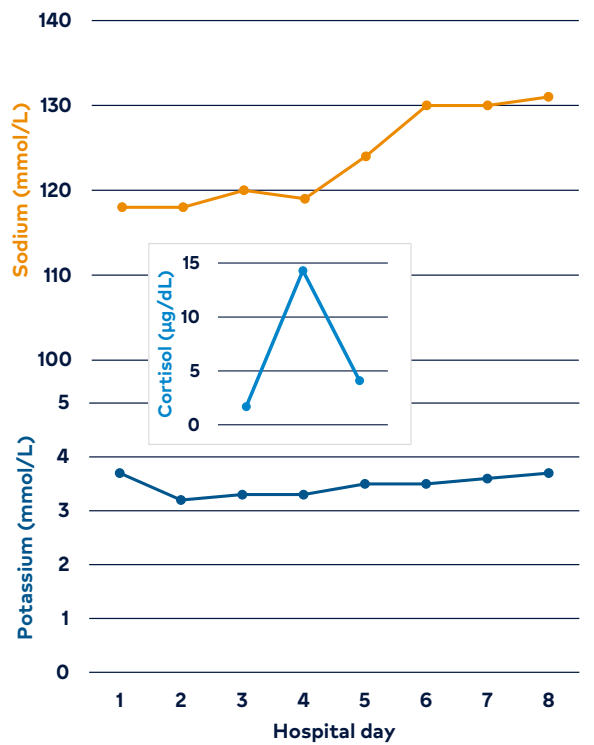

Figure 2. Levels of sodium and potassium during hospitalization. Cortisol levels on hospital days 3-5 shown in the insert. Day 1: Administration of 0.9\% normal saline; Day 2: Water restriction and salt tablets; Day 3: Cosyntropin stimulation; Day 4: Treatment with hydrocortisone.

than or equal to $13 \%) .{ }^{5}$ The exact mechanism of primary adrenal insufficiency is unclear. However, secondary adrenal insufficiency is thought to be caused by inflammation of the pituitary gland or pituitary stalk due to lymphocytic infiltration seen mostly with the use of the anti-CTLA-4 ICI, such as ipilimumab, when compared with anti-PD-1/PDL-1 therapies. A possible explanation for this is that the expression of CTLA-4 in the pituitary gland acts as an autoantigen. ${ }^{8,9}$ The degree of pituitary gland inflammation varies and may even be unnoticeable on imaging. ${ }^{6,10}$ Therefore, patients may remain asymptomatic despite sustaining injury to the pituitary following $\mathrm{ICl}$ treatment.

In this case, the patient completed a full course of 4 rounds of weekly treatment with ipilimumab/nivolumab. He was doing relatively well and could perform his activities of daily living including driving to his appointments, suggesting an intact hypothalamic pituitary adrenal axis. However, he became symptomatic immediately after he received his first dose of radiation therapy. He suddenly experienced generalized weakness, particularly proximal muscle weakness, and was unable to feed himself. $\mathrm{He}$ also experienced headaches, nausea and presyncopal episodes before presentation to the hospital. Because his symptoms started immediately after radiation therapy, it is likely that further injury to his pituitary gland, previously exposed to ICls, occurred. That is, the effects of radiation and $\mathrm{ICl}$ therapies on the pituitary gland were synergistic. Although he was clinically asymptomatic prior to radiation therapy, he was on the verge of experiencing pituitary malfunction that only materialized after radiation therapy.

The patient experienced secondary adrenal insufficiency caused by hypopituitarism as his FSH, TSH and ACTH levels were low. His cortisol level responded to cosyntropin stimulation. Since $\mathrm{ICl}$-induced hypopituitarism can occur weeks to months after initiation of therapy, it is possible that his symptoms were caused by $\mathrm{ICl}$ treatment alone, irrespective of additional radiation therapy. ${ }^{6}$ However, his sudden onset of symptoms following radiation is suggestive of a dual-hit cause since the initiation of dabrafenib and trametinib occurred only after the patient became symptomatic. It is also unlikely that these medications caused his symptoms since these two drugs have not been associated with hypopituitarism.

\section{Conclusion}

This case highlights a rare situation in which additional insult, in the form of radiation therapy, to the pituitary gland previously exposed to ICls led to life-threatening hypopituitarism and secondary adrenal insufficiency. As highlighted here, patients treated with ICls may re- 
main asymptomatic clinically but will be on the verge of experiencing life-threatening crises that can occur if exposed to a second form of injury to the pituitary gland. Thus, for patients treated with ICls, particularly with ipilimumab/ nivolumab, a washout period may be considered before initiating other forms of therapies, such as radiation to the head/neck regions, which is capable of causing further injury to the pituitary. Otherwise, prophylactic lowdose hydrocortisone may be initiated in cases where radiation therapy must not be delayed. To date, the use of hydrocortisone for prophylaxis in such scenarios has not been specifically discussed in the literature. Instead, routine monitoring of pituitary function has been emphasized. Although such monitoring can detect early pituitary dysfunction, it may not be helpful in other cases where abrupt dysfunction is caused by a second insult, as described in this case. As more cases like this are described in the literature, we remain optimistic that the consensus on preventing and managing such adverse effects caused by combined ICls/radiation will be updated accordingly.

\section{Conflicts of Interest}

The authors declare they have no conflicts of interest.

The authors are employees of North Florida Regional Medical Center, a hospital affiliated with the journal's publisher.

This research was supported (in whole or in part) by HCA Healthcare and/or an HCA Healthcare affiliated entity. The views expressed in this publication represent those of the author(s) and do not necessarily represent the official views of HCA Healthcare or any of its affiliated entities.

\section{Author Affiliations}

1. University of Central Florida, College of Medicine/North Florida Regional Medical Center, Gainesville, FL

\section{References}

1. Abril-Rodriguez G, Ribas A. SnapShot: immune checkpoint inhibitors. Cancer Cell. 2017;31(6):848848.e1. https://doi.org/10.1016/i.ccell.2017.05.010

2. Michot JM, Bigenwald C, Champiat S, et al. Immune-related adverse events with immune checkpoint blockade: a comprehensive review. Eur J Cancer. 2016;54:139-148. https://doi. org/10.1016/j.ejca.2015.11.016
3. Hodi FS, Chiarion-Sileni V, Gonzalez R, et al. Nivolumab plus ipilimumab or nivolumab alone versus ipilimumab alone in advanced melanoma (CheckMate 067): 4-year outcomes of a multicentre, randomised, phase 3 trial. Lancet Oncol. 2018;19(11):1480-1492. https://doi.org/10.1016/ s1470-2045(18)30700-9

4. Postow MA, Sidlow R, Hellmann MD. Immune-related adverse events associated with immune checkpoint blockade. N Engl J Med. 2018;378(2):158-168. https://doi.org/10.1056/neimra1703481

5. Puzanov I, Diab A, Abdallah K, et al. Managing toxicities associated with immune checkpoint inhibitors: consensus recommendations from the Society for Immunotherapy of Cancer (SITC) Toxicity Management Working Group. $J$ Immunother Cancer. 2017;5(1):95. https://doi. org/10.1186/s40425-017-0300-z

6. Lupi I, Brancatella A, Cosottini M, et al. Clinical heterogeneity of hypophysitis secondary to PD-1/PD-L1 blockade: insights from four cases. Endocrinol Diabetes Metab Case Rep. 2019;2019:19-0102. https://doi.org/10.1530/edm19-0102

7. Del Rivero J, Cordes LM, Klubo-Gwiezdzinska J, Madan RA, Nieman LK, Gulley JL. Endocrine-related adverse events related to immune checkpoint inhibitors: proposed algorithms for management. Oncologist. 2020;25(4):290-300. https://doi.org/10.1634/theoncologist.2018-0470

8. Ueda H, Howson JM, Esposito L, et al. Association of the T-cell regulatory gene CTLA4 with susceptibility to autoimmune disease. Nature. 2003;423(6939):506-511. https://doi.org/10.1038/ nature01621

9. Iwama S, De Remigis A, Callahan MK, Slovin SF, Wolchok JD, Caturegli P. Pituitary expression of CTLA-4 mediates hypophysitis secondary to administration of CTLA-4 blocking antibody. Sci Trans/ Med. 2014;6(230):230ra45. https://doi. org/10.1126/scitranslmed.3008002

10. Chodakiewitz Y, Brown S, Boxerman JL, Brody JM, Rogg JM. Ipilimumab treatment associated pituitary hypophysitis: clinical presentation and imaging diagnosis. Clin Neurol Neurosurg. 2014;125:125-130. https://doi.org/10.1016/j.clineuro.2014.06.011 